\title{
Optimal treatment sequence for targeted immune modulators for the treatment of moderate to severe ulcerative colitis
}

Lisa M Bloudek, PharmD, MS; Rajshree Pandey, PhD, MPH; Katherine Fazioli; Daniel A Ollendorf, PhD; and Josh J Carlson, PhD

\section{What is already known about this subject}

- Targeted immune modulators (TIMS), consisting of several monoclonal antibodies and 1 oral Janus kinase inhibitor, have shown effectiveness in inducing remission and response in patients with moderate to severe ulcerative colitis and are approved by the FDA for this indication.

- Patients may try multiple TIMs, and currently there are no biomarkers or prognostic factors to guide choice of treatment sequence.

- In 2020, the Institute for Clinical and Economic Review (ICER) conducted a review of TIMs for the treatment of moderate to severe ulcerative colitis as individual agents but did not address the cost-effectiveness of various treatment sequences.

\section{What this study adds}

- This study leveraged the ICERdeveloped cost-effectiveness model to investigate optimal treatment sequencing of TIMs for the treatment of moderate to severe ulcerative colitis based on 4 objectives: maximizing net health benefit, minimizing cost, maximizing QALYs, or convenience.

- Sequences that were most likely to maximize net health benefit and minimize cost began with the infliximab biosimilar product, then tofacitinib, adalimumab, or vedolizumab were used as subsequent treatment for those with lack of response or on discontinuation.

- Sequences most likely to maximize QALYs began with ustekinumab, followed by vedolizumab, tofacitinib, and adalimumab.

\begin{abstract}
Author affiliations
Lisa M Bloudek, PharmD, MS, and Josh J Carlson, PhD, Comparative Health Outcomes, Policy \& Economics (CHOICE) Institute, University of Washington School of Pharmacy, Seattle. Rajshree Pandey, $\mathrm{PhD}, \mathrm{MPH}$, and Katherine Fazioli, Institute for Clinical and Economic Review, Boston, MA. Daniel A Ollendorf, PhD, Center for the Evaluation of Value and Risk in Health, Tufts Medical Center, Boston, MA.
\end{abstract}

AUTHOR CORRESPONDENCE:

Lisa Bloudek, 206.375.8471;

lbloudek@uw.edu

J Manag Care Spec Pharm 2021;27(8):1046-55

Copyright $\odot 2021$, Academy of Managed Care Pharmacy. All rights reserved.

response without achieving remission, clinical remission, and death. Efficacy of TIMs were informed by the ICER-conducted network meta-analysis. Up to 3 treatments were modeled in a sequence that consisted of 2 different TIMs followed by conventional treatment. Sequences were ranked according to each objective. NHB was calculated using a threshold of $\$ 150,000$ per QALY gained. Probabilistic sensitivity analysis (PSA) was undertaken to estimate the probability of each sequence having the highest NHB rank under each objective.

RESULTS: 21 possible sequences were evaluated in the base case. Two attempts 
at conventional treatment represented the lowest cost option and, while yielding the fewest QALYs, resulted in the highest NHB. None of the sequences had an incremental cost per QALY below $\$ 150,000$ relative to 2 attempts with conventional treatment, so the resulting NHB was negative for all sequences. The sequence with the highest NHB was infliximab-dyyb followed by tofacitinib $(-0.116)$. This regimen also had the lowest incremental costs $(\$ 37,266)$. For orally and subcutaneously administered TIMs, the sequence of golimumabtofacitinib had the highest NHB (-0.344). Ustekinumab-vedolizumab was the top-ranked sequence as measured by QALY maximization ( 0.172 incremental QALYs) but also had the highest total incremental cost $(\$ 166,094)$. Results of the PSA were consistent with deterministic rankings for the top-ranking sequences but also showed that the top 2 or 3 regimens were often close together.

CONCLUSIONS: Based on the results of this analysis, the optimal sequence of TIMs as measured by NHB and cost minimization was infliximab or biosimilars as first-line treatment, then moving to tofacitinib, adalimumab, or vedolizumab. Sequences that generated the most QALYs began with ustekinumab, followed by vedolizumab, tofacitinib, and adalimumab.

Ulcerative colitis is a chronic, immune-mediated, inflammatory condition of the large intestine and rectum that affects approximately 900,000 people in the United States. ${ }^{1}$ The disease follows a relapsing-remitting course characterized by bleeding, diarrhea, and abdominal pain. The majority of cases are mild to moderate in severity. ${ }^{2}$ Active moderate to severe disease is associated with symptoms of stool frequency, fecal incontinence, weight loss, fatigue, pain, and bleeding, which are frequently incapacitating in adults, with the added potential for irreversible negative impact on growth and development for children with ulcerative colitis. ${ }^{3,4}$ The disease imparts significant direct and indirect costs, including lost productivity, psychosocial and family impact, in addition to the substantial impact on quality of life. ${ }^{5,6}$

Mild to moderate ulcerative colitis is often managed with dietary modification, corticosteroids, and systemic immunomodulators such as azathioprine. ${ }^{7}$ For those with moderate to severe disease, oral corticosteroids may induce remission, but repeated courses and continued use of corticosteroids for maintenance of remission is not recommended because of the potential for serious adverse events. ${ }^{8}$ Several monoclonal antibodies and 1 oral Janus kinase (JAK) inhibitor have shown effectiveness in inducing remission and response in patients with moderate to severe ulcerative colitis, as well as maintaining response/ remission over the long term, and are approved by the US Food and Drug Administration (FDA) for 1 or both of these indications.
For patients who continue to experience debilitating and potentially life-threatening symptoms of ulcerative colitis despite maximal medical treatment, surgical removal of the colon (colectomy) is an option. ${ }^{8}$ While curative, colectomy does not modify the underlying inflammatory cause of ulcerative colitis. Extraintestinal manifestations, such as sclerosing cholangitis and arthropathy may still occur.,10 The disease may reoccur elsewhere in the gastrointestinal tract as Crohn disease.11,12 Even if curative for ulcerative colitis, colectomy is associated with short- and long-term complications of the ileal pouchitis and negative consequences such as infertility. ${ }^{13,14}$ For these reasons, multiple rounds of advanced pharmaceutical treatment are often attempted before consideration of colectomy.

The approved monoclonal antibodies and JAK inhibitor, which as a group is referred to here as targeted immune modulators (TIMs), include antitumor necrosis factor (TNF) inhibitors (adalimumab, golimumab, infliximab, and infliximab biosimilars); a JAK inhibitor (tofacitinib); an IL-12/23 inhibitor (ustekinumab); and a gut-specific a $4 \beta 7$ integrin inhibitor (vedolizumab). Although adalimumab biosimilars are approved, none were marketed or available to patients at the time of analysis.

In a recent review of the comparative effectiveness of TIMs for the treatment of moderate to severe ulcerative colitis conducted by the Institute for Clinical and Economic Review (ICER), the TIMs were found to be largely similar with regards to efficacy and safety. The ICER-conducted network meta-analysis (NMA) resulted in lower efficacy of induction and maintenance for adalimumab compared with other TIMs in the biologic-naive and biologic-experienced populations. This indirect evidence is further supported by direct evidence from a head-to-head study that showed substantial and statistically significant differences in remission and response in favor of vedolizumab vs adalimumab. ${ }^{15}$ While similar in efficacy, the TIMs were wide-ranging in annual costs. ${ }^{15}$ These results are based on average gains across patients enrolled in randomized controlled trials.

In clinical practice, response to therapy is highly heterogeneous, and unfortunately, there are no known biomarkers or predictive factors to inform TIM treatment selection. ${ }^{16}$ This leads to a process of trial and error in search of the right drug for each patient. ${ }^{17}$ Guidelines from the American Gastroenterology Association list all agents except tofacitinib as potential initial treatment options, with sparse recommendations based on low-quality evidence for choice of any given subsequent TIM over another. ${ }^{8,18}$ In the current practice landscape, insurance coverage and step edit requirements often influence the selection of TIM agents, with formulary placement driven by cost and contractual 


\section{FIGURE 1 Model Structure}

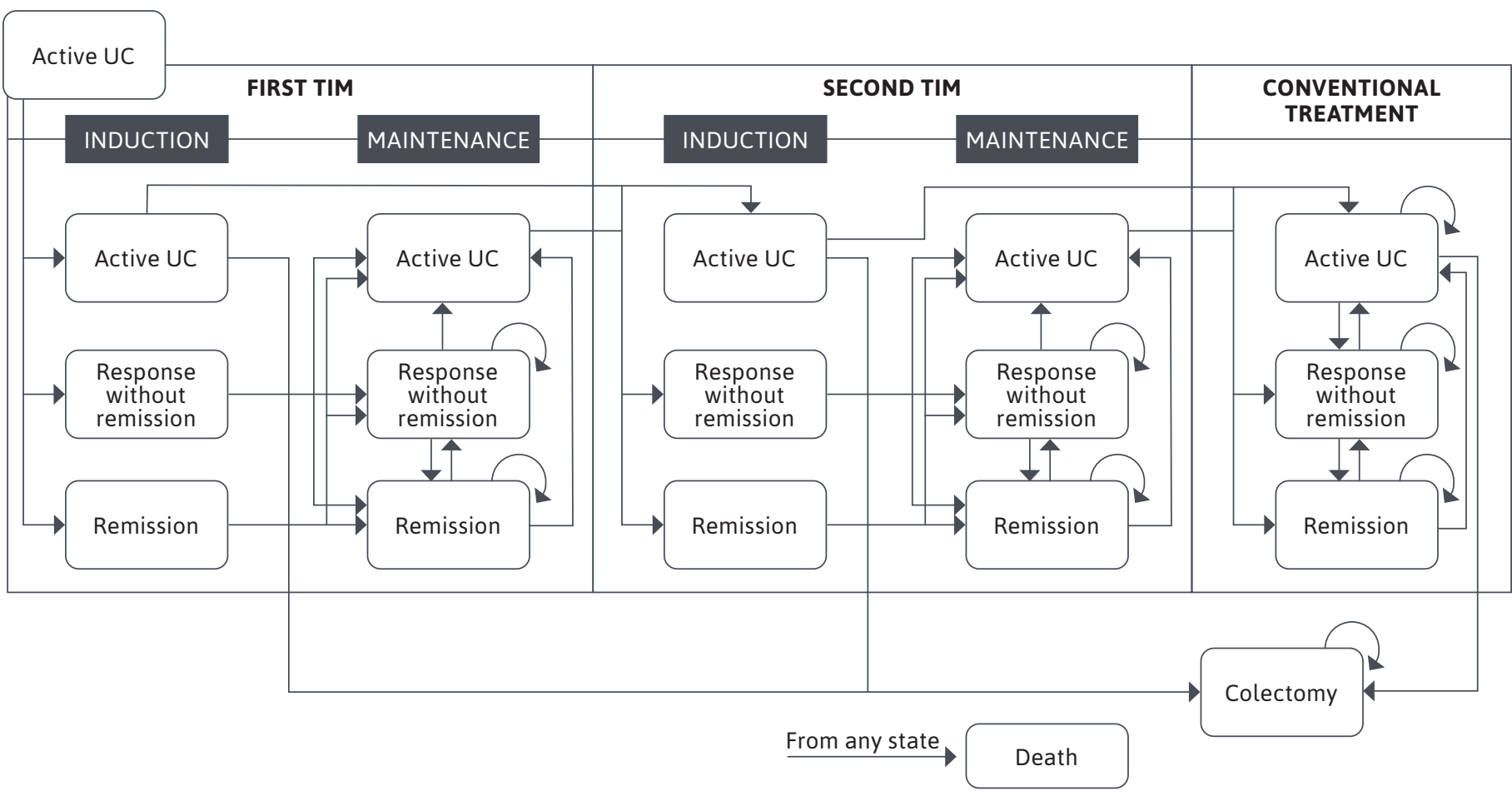

TIM= targeted immune modulator; UC=ulcerative colitis.

arrangements rather than by efficacy or cost-effectiveness and often not in concordance with clinical guidelines. ${ }^{15}$

Given that there is no reliable way to predict which individual patient will benefit from one TIM or another, the TIMs for moderate to severe ulcerative colitis represent a scenario in which the preferred option is to try the most cost-effective option first, a combination of relative efficacy and relative cost. The 2020 ICER review assessed the costeffectiveness of TIMs vs conventional treatment, informed by the placebo arms of clinical trials, separately in biologicnaive and biologic-experienced populations. However, the scope of this review did not address optimal sequencing of treatments.

We sought to extend the ICER framework to investigate the cost-effectiveness of each treatment sequence informed by various objectives: net health benefit (NHB), quality-adjusted life-year (QALY) maximization, cost minimization, or convenience. NHB is a metric of the impact of a treatment on total population health that combines health gains for patients who receive the treatment with the health opportunity cost experienced by other patients, under the assumption that opportunity costs can be converted into the health lost using a willingness-to-pay (WTP) threshold. ${ }^{19}$ A positive NHB indicates that the treatment is cost-effective at the chosen WTP threshold. An NHB approach has been used to inform technology assessments where treatment sequencing is an issue. ${ }^{20,21}$ Choosing a sequence that maximizes the NHB may be of value to inform formulary decision makers, physicians, and patients; however, prioritization of treatment sequences may also be driven by other measures of value.

Some decision makers who place a higher value on clinical benefit and a lower value on relative cost may look for the QALY-maximizing sequence, or if clinical benefit across TIMs is viewed to be relatively similar, a higher value could be placed on relative cost (eg, cost minimizing). The American Gastroenterology Association practice guidelines state that patients who place higher value on the convenience of self-administered subcutaneous injection, and a lower value on the relative efficacy of medications, may reasonably choose a less effective self-administered treatment option as an alternative to a more effective intravenously 


\section{TABLE 1 Incremental Cost, QALYs, and NHB of TIMs Compared With Conventional Treatment}

\begin{tabular}{|c|c|c|c|c|c|c|}
\hline & Incremental cost & $\begin{array}{l}\text { Incremental cost } \\
\text { minimization rank }\end{array}$ & Incremental QALYs & $\begin{array}{l}\text { Incremental QALY } \\
\text { maximizing rank }\end{array}$ & NHB & $\begin{array}{c}\text { Incremental NHB } \\
\text { rank }\end{array}$ \\
\hline \multicolumn{7}{|c|}{ Biologic-naive population } \\
\hline Infliximab-dyyb & $\$ 10,859$ & 1 & 0.071 & 2 & 0.00 & 1 \\
\hline Infliximab-abda & $\$ 11,174$ & 2 & 0.071 & 2 & 0.00 & 2 \\
\hline Infliximab & $\$ 12,347$ & 3 & 0.071 & 2 & -0.01 & 3 \\
\hline Golimumab & $\$ 38,135$ & 4 & 0.026 & 4 & -0.23 & 4 \\
\hline Adalimumab & $\$ 41,057$ & 5 & 0.022 & 5 & -0.25 & 5 \\
\hline Vedolizumab & $\$ 53,646$ & 6 & 0.068 & 3 & -0.29 & 6 \\
\hline Ustekinumab & $\$ 124,927$ & 7 & 0.108 & 1 & -0.72 & 7 \\
\hline \multicolumn{7}{|c|}{ Biologic-experienced population } \\
\hline Tofacitinib & $\$ 26,430$ & 1 & 0.053 & 3 & -0.12 & 1 \\
\hline Adalimumab & $\$ 30,725$ & 2 & 0.017 & 4 & -0.19 & 2 \\
\hline Vedolizumab & $\$ 42,657$ & 3 & 0.054 & 2 & -0.23 & 3 \\
\hline Ustekinumab & $\$ 70,532$ & 4 & 0.057 & 1 & -0.41 & 4 \\
\hline
\end{tabular}

$\mathrm{NHB}=$ net health benefit; $\mathrm{QALY}=$ quality-adjusted life-year; TIM=targeted immune modulator.

administered option. ${ }^{18}$ Therefore, identifying the treatment sequence with the greatest NHB among self-administered options may be informative for decision making in some circumstances. The objective of this study was to assess the clinical and economic impact of ulcerative colitis treatment sequences informed by various objectives, including the NHB approach.

\section{Methods}

The structure and inputs of the ICER model have been presented previously. ${ }^{15}$ Briefly, the model was constructed as a Markov model with 8-week cycles over a lifetime time horizon. The model was developed in Microsoft Excel.

Health states consisted of active moderate to severe ulcerative colitis, clinical response without achieving remission, clinical remission, and death (Figure 1). The base-case analysis used a US payer perspective, considering only direct health care costs. Patients entered the model in the active health state and were treated with a TIM or conventional treatment. Treatment was aimed at inducing clinical response or complete remission in the short-term (induction phase, 6-14 weeks) and maintaining response or remission in the longer term (maintenance phase). In alignment with clinical practice, those who achieved clinical response (with or without clinical remission) at the end of induction were assumed to remain on treatment as maintenance, often at a lower dose than administered during the induction phase. Those with no response or remission initiated treatment with a subsequent TIM. Those with no response or remission on the subsequent TIM initiated treatment with conventional treatment for the remainder of the model time horizon.

A constant per-cycle probability of colectomy was applied to the active ulcerative colitis health state. Drug acquisition cost was based on wholesale acquisition cost (WAC) for subcutaneously administered products and average sales price plus $9.5 \%$ for intravenously administered products. Average discounts sourced for SSR Health were applied to WAC to estimate net prices. Other costs included cost of administration, colectomy and colectomy complications, severe infection, hospitalization, outpatient visits, and emergency department visits. Health state and health care resource use costs from the literature were inflated to 2019 US dollars.

Drug costs were updated to reflect prices as of May 2020. QALYs were derived based on time spent in health states of nonresponse, response without remission, response with remission, and postcolectomy. A utility decrement was applied for perioperative colectomy complications, chronic pouchitis postcolectomy, and severe infection. All costs and outcomes were discounted at an annual rate of 3\%. Efficacy of TIMs in the biologic-naive and biologic-experienced populations were informed by the ICER-conducted NMAs of TIMs in each of these populations. 


\begin{tabular}{|c|c|c|c|c|c|c|c|c|}
\hline Sequence & Cost & $\Delta$ Cost & $\begin{array}{c}\text { Incremental } \\
\text { cost } \\
\text { minimizing } \\
\text { rank }\end{array}$ & QALYs & $\triangle Q A L Y s$ & $\begin{array}{c}\text { Incremental } \\
\text { QALY } \\
\text { maximizing } \\
\text { rank }\end{array}$ & NHB & $\begin{array}{c}\text { Incremental } \\
\text { NHB rank }\end{array}$ \\
\hline Infliximab-dyyb > tofacitinib & $\$ 414,580$ & $\$ 37,266$ & 1 & 15.650 & 0.133 & 10 & -0.116 & 1 \\
\hline Infliximab > tofacitinib & $\$ 416,067$ & $\$ 38,753$ & 2 & 15.650 & 0.133 & 9 & -0.125 & 2 \\
\hline Infliximab-dyyb > adalimumab & $\$ 417,938$ & $\$ 40,625$ & 3 & 15.619 & 0.102 & 13 & -0.169 & 3 \\
\hline Infliximab > adalimumab & $\$ 419,425$ & $\$ 42,112$ & 4 & 15.619 & 0.102 & 12 & -0.178 & 4 \\
\hline Infliximab-dyyb > vedolizumab & $\$ 429,859$ & $\$ 52,546$ & 5 & 15.652 & 0.135 & 7 & -0.215 & 5 \\
\hline Infliximab > vedolizumab & $\$ 431,346$ & $\$ 54,033$ & 6 & 15.652 & 0.135 & 6 & -0.225 & 6 \\
\hline Golimumab $>$ tofacitinib & $\$ 442,247$ & $\$ 64,934$ & 7 & 15.606 & 0.089 & 17 & -0.344 & 7 \\
\hline Adalimumab $>$ tofacitinib & $\$ 445,204$ & $\$ 67,891$ & 8 & 15.602 & 0.085 & 20 & -0.368 & 8 \\
\hline Infliximab-dyyb > ustekinumab & $\$ 457,115$ & $\$ 79,802$ & 10 & 15.653 & 0.136 & 5 & -0.396 & 9 \\
\hline Golimumab > adalimumab & $\$ 445,650$ & $\$ 68,336$ & 9 & 15.575 & 0.058 & 21 & -0.397 & 10 \\
\hline Vedolizumab $>$ tofacitinib & $\$ 457,389$ & $\$ 80,075$ & 11 & 15.647 & 0.130 & 11 & -0.403 & 11 \\
\hline Infliximab > ustekinumab & $\$ 458,602$ & $\$ 81,289$ & 13 & 15.653 & 0.136 & 4 & -0.406 & 12 \\
\hline Golimumab > vedolizumab & $\$ 457,748$ & $\$ 80,434$ & 12 & 15.608 & 0.091 & 16 & -0.445 & 13 \\
\hline Vedolizumab > adalimumab & $\$ 460,750$ & $\$ 83,436$ & 15 & 15.617 & 0.100 & 14 & -0.456 & 14 \\
\hline Adalimumab > vedolizumab & $\$ 460,724$ & $\$ 83,410$ & 14 & 15.604 & 0.087 & 19 & -0.469 & 15 \\
\hline Golimumab > ustekinumab & $\$ 485,400$ & $\$ 108,086$ & 16 & 15.610 & 0.093 & 15 & -0.628 & 16 \\
\hline Adalimumab > ustekinumab & $\$ 488,410$ & $\$ 111,096$ & 17 & 15.605 & 0.089 & 18 & -0.652 & 17 \\
\hline Vedolizumab > ustekinumab & $\$ 499,960$ & $\$ 122,646$ & 18 & 15.651 & 0.134 & 8 & -0.684 & 18 \\
\hline Ustekinumab > tofacitinib & $\$ 528,315$ & $\$ 151,001$ & 19 & 15.686 & 0.170 & 2 & -0.837 & 19 \\
\hline Ustekinumab > adalimumab & $\$ 531,636$ & $\$ 154,323$ & 20 & 15.656 & 0.139 & 3 & -0.889 & 20 \\
\hline Ustekinumab > vedolizumab & $\$ 543,407$ & $\$ 166,094$ & 21 & 15.688 & 0.172 & 1 & -0.936 & 21 \\
\hline Conventional treatment & $\$ 377,313$ & - & & 15.52 & - & & - & \\
\hline
\end{tabular}

$\Delta=$ difference; $N H B=$ net health benefit; $Q A L Y=$ quality-adjusted life-year; $T I M=$ targeted immune modulator.

This study had 2 primary phases: an interventionranking exercise to construct the sequences, followed by an evaluation phase comparing the chosen sequences.

\section{PHASE 1: INDIVIDUAL LINES OF THERAPY}

In the original ICER analysis, the treatment pathway for TIMs and conventional treatment consisted of initial treatment, subsequent treatment, and then conventional treatment. For phase 1, we removed subsequent treatment from the pathways of TIMs and conventional treatment to remove any influence of subsequent TIMs on the evaluation of initial treatment-an approach used by other health technology assessment agencies..$^{20,21}$ We then calculated the incremental NHB of each TIM in a biologic-naive population and biologic-experienced population, compared with conventional treatment to rank order the TIMs from highest to lowest incremental NHB. Incremental NHB was calculated using a threshold of $\$ 150,000$ per QALY gained using the following equation ${ }^{19}$ :

$$
\text { Incremental QALYs- } \frac{\text { Incremental cost }}{\text { Threshold }}
$$

Thresholds of $\$ 50,000$ and $\$ 100,000$ per QALY were explored as alternative scenarios. We also ranked the interventions according to incremental QALYs generated (QALY maximizing) and lowest incremental total costs (cost minimizing).

Finally, the route of administration for TIMs varies. Golimumab and adalimumab are administered subcutaneously; ustekinumab is administered intravenously during induction and subcutaneously for maintenance; tofacitinib is taken orally; and infliximab and vedolizumab are 


\section{TABLE 3 Results of PSA for Top 5 Sequences Compared With Conventional Treatment}

\begin{tabular}{c|l|c|l|l|l}
\multicolumn{2}{|c|}{ Incremental cost minimizing } & \multicolumn{3}{c|}{ Incremental QALY maximizing } & \multicolumn{2}{c}{ Incremental NHB maximizing } \\
\hline $31 \%$ & Infliximab-dyyb-tofacitinib & $23 \%$ & Ustekinumab-vedolizumab & $24 \%$ & Infliximab-dyyb-tofacitinib \\
\hline $27 \%$ & Infliximab-dyyb-adalimumab & $19 \%$ & Ustekinumab-tofacitinib & $22 \%$ & Infliximab-dyyb-vedolizumab \\
\hline $16 \%$ & Infliximab-dyyb-vedolizumab & $17 \%$ & Ustekinumab-adalimumab & $19 \%$ & Infliximab-dyyb-adalimumab \\
\hline $10 \%$ & Infliximab-tofacitinib & $11 \%$ & Vedolizumab-ustekinumab & $15 \%$ & Infliximab-dyyb-ustekinumab \\
\hline $8 \%$ & Infliximab-adalimumab & $7 \%$ & Infliximab-dyyb-ustekinumab & $7 \%$ & Infliximab-adalimumab \\
\hline
\end{tabular}

$\mathrm{NHB}=$ net health benefit; $P S A=$ probabilistic sensitivity analysis; $Q A L Y=$ quality-adjusted life-year.

administered via intravenous infusion. Because of convenience or other factors, some patients may not prefer an intravenous route of administration. For this reason, we also investigated which treatment sequence of orally or subcutaneously administered TIMs led to the highest NHB.

\section{PHASE 2: SEQUENCE RANKING}

In phase 2 , we reinstated the subsequent TIM structure in order to calculate the outcomes for each sequence of TIMs. We compared sequences within each objective, ie, NHB, QALY maximizing, and cost minimizing vs a sequence of 2 attempts at conventional treatment with transition probabilities for biologic-naive patients in the first conventional treatment line and transition probabilities for biologicexperienced patients in the second conventional treatment line. Finally, we compared the highest-ranking sequence for each objective to each other and conventional treatment. It was assumed that patients would not use a specific TIM twice (ie, in first-line and second-line treatments).

Tofacitinib was not included as an option for initial TIM treatment because of labeling that limits use to after failure of an anti-TNF inhibitor. ${ }^{22}$ Infliximab and golimumab were not included as an option for subsequent treatment after initial TIM in the original ICER analysis because of the lack of randomized controlled trial data in a biologicexperienced population, which would allow for inclusion in the biologic-experienced NMA. However, infliximab is commonly used following other TIMs in real-world practice. ${ }^{23,24}$ We conducted a scenario analysis where infliximab was included as an option as second-line TIM. Efficacy in a biologic-experienced (eg, second line) population is often reduced compared with a biologic-naive population. The exact magnitude of this difference is highly variable across TIMs. For this scenario, we assumed a 20\% reduction in the efficacy of infliximab in inducing response/remission and no difference in maintenance efficacy.

\section{OUTCOMES}

The outcomes of our analysis included incremental $\mathrm{NHB}$, costs, and QALYs, comparing across the treatment sequences, with ranking informed by each objective. Probabilistic sensitivity analysis (PSA) was undertaken to estimate the probability of each treatment sequence having the highest NHB rank, QALY maximizing rank, and cost minimizing rank. PSA was implemented by simultaneously varying all model parameters over 1,000 simulations using parameter distributions and NMA coda samples as in the original ICER evaluation.

\section{Results}

\section{PHASE 1: INDIVIDUAL LINES OF THERAPY}

The incremental NHB, incremental cost minimizing, and incremental QALY maximizing ranking of each TIM compared with conventional treatment in the biologic-naive and biologic-experienced populations are presented in Table 1. None of the TIMs had an incremental cost per QALY below $\$ 150,000$ compared with 2 attempts at conventional treatment in the base-case analysis. As such, the resulting NHB was negative for all TIMs at the $\$ 150,000$ per QALY threshold. In the biologic-naive population, infliximab-dyyb, an infliximab biosimilar, had the least negative (ie, most favorable) NHB. Ustekinumab resulted in the maximum incremental QALYs, followed by infliximab and its biosimilars. In the biologic-experienced population, tofacitinib had the least negative NHB. However, tofacitinib ranked third behind ustekinumab and vedolizumab for incremental QALYs. Among orally or subcutaneously administered TIMs in the biologic-naive population (ie, golimumab, adalimumab, and ustekinumab), golimumab had the least negative NHB, but ustekinumab resulted in the highest QALYs. 


\begin{tabular}{|c|c|c|c|c|c|c|c|c|}
\hline Sequence & Cost & $\Delta$ Cost & $\begin{array}{c}\text { Incremental } \\
\text { cost } \\
\text { minimizing } \\
\text { rank }\end{array}$ & QALYs & $\triangle Q A L Y s$ & $\begin{array}{l}\text { Incremental } \\
\text { QALY } \\
\text { maximizing } \\
\text { rank }\end{array}$ & NHB & $\begin{array}{c}\text { Incremental } \\
\text { NHB rank }\end{array}$ \\
\hline Golimumab-infliximab & $\$ 424,373$ & $\$ 47,060$ & 5 & 15.570 & 0.053 & 26 & -0.261 & 7 \\
\hline Adalimumab-infliximab & $\$ 427,307$ & $\$ 49,994$ & 6 & 15.566 & 0.049 & 27 & -0.284 & 8 \\
\hline Vedolizumab-infliximab & $\$ 439,759$ & $\$ 62,446$ & 9 & 15.611 & 0.095 & 18 & -0.322 & 9 \\
\hline Ustekinumab-infliximab & $\$ 510,921$ & $\$ 133,607$ & 23 & 15.651 & 0.134 & 10 & -0.756 & 23 \\
\hline
\end{tabular}

$\triangle$ =difference; $N H B=$ net health benefit; $Q A L Y=$ quality-adjusted life-year.

\section{PHASE 2: SEQUENCE RANKING}

Sequence Ranking Based on NHB. Table 2 compares all possible TIM sequences to a sequence of 2 attempts at conventional treatment. No sequences had a positive NHB, suggesting that 2 attempts at conventional treatment resulted in higher total population health relative to any of the TIM sequences. Infliximab-abda was assumed to have equal efficacy to that of infliximab and infliximab-dyyb, with a marginally higher net price than infliximab-dyyb, and thus always ranked 1 place behind infliximab-dyyb (data not shown). The sequence with the highest NHB was infliximab-dyyb followed by tofacitinib (infliximabdyyb-tofacitinib). The sequence with the lowest NHB was ustekinumab-vedolizumab. For orally and subcutaneously administered TIMs, the sequence of golimumab-tofacitinib had the highest NHB.

Sequence Ranking Based on Cost Minimization. The 5 sequences with the lowest incremental cost compared with conventional treatment are also presented in Table 2. This ranking was identical to the ranking based on NHB. Incremental costs ranged from $\$ 37,266$ for infliximab-dyybtofacitinib to $\$ 166,094$ for ustekinumab-vedolizumab.

Sequence Ranking Based on QALY Maximization. Although having a higher NHB, the sequence of infliximab-tofacitinib did not result in the greatest number of QALYs gained. All 5 sequences with the greatest QALY gains over conventional treatment contained ustekinumab. Ustekinumab-vedolizumab, ustekinumab-tofacitinib, and ustekinumab-adalimumab held the top 3 rankings, respectively, followed by infliximab-dyyb-ustekinumab and infliximab-ustekinumab. The incremental QALY gain with ustekinumab-vedolizumab over infliximab-dyyb-tofacitinib was 0.039 at an incremental cost of $\$ 128,828$, yielding an incremental cost per QALY of $\$ 3,319,214$.

\section{PROBABILISTIC SENSITIVITY ANALYSIS}

Table 3 presents the top 5 sequences as measured by lowest cost, greatest QALYs, or greatest NHB as ranked by results of the PSA. Results of the PSA were generally consistent with deterministic estimates for the top ranking sequence under each objective, with some differences in ranking the second and onward. In all cases, the top 3 sequences listed in Table 3 resulted in optimal outcomes in at least $60 \%$ of PSA iterations, and the top 2 covered at least $40 \%$ of PSA iterations.

\section{SCENARIO ANALYSIS}

NHB rankings using WTP thresholds of $\$ 50,000$ per QALY gained and $\$ 200,000$ per QALY gained resulted in rankings that were identical to the base case.

The scenario where infliximab is used as second-line treatment after adalimumab, golimumab, ustekinumab, or vedolizumab is presented in Table 4 . Assuming a $20 \%$ reduction in induction efficacy for a biologic-experienced population relative to a biologic-naive population, none of the sequences with infliximab as second-line treatment were in the top 5 rankings as measured by NHB, cost minimization, or QALY maximization. This finding is maintained for NHB and cost minimization if the $20 \%$ efficacy penalty for infliximab in a biologic-experienced population is removed. However, once removed, the ustekinumabinfliximab ranks third for QALY maximization (incremental gain of 0.142).

\section{Discussion}

In the previous evaluation of TIMs for the treatment of ulcerative colitis conducted by ICER, no TIMs were found to be cost-effective relative to conventional treatment in the biologic-naive or biologic-experienced populations, with incremental cost per QALY gained ranging from $\$ 186,000$ for infliximab-dyyb in the biologic-naive population to 
$\$ 1,885,000$ for adalimumab in the biologic-experienced population. ${ }^{15}$ Results were driven by the high cost of TIMs and relatively modest efficacy relative to placebo in inducing and maintaining response.

In this study, we leveraged the ICER ulcerative colitis model framework developed for the evaluation of the long-term cost-effectiveness of individual TIMs and reframed it to consider optimal treatment sequences based on different objectives, ie, NHB, cost minimization, QALY maximization, or convenience for all TIMs. These sequences were constructed by ranking the interventions in the biologic-naive and biologic-experienced populations by each objective and then combining the top ranked interventions without rechallenge. The sequences were then compared with a sequence of conventional treatment alone. The top sequences for each objective were also compared with each other.

Results of our analysis showed that the top-ranking sequences as measured by NHB or cost minimization started with infliximab or biosimilars as first-line treatment, then moved to tofacitinib, adalimumab, or vedolizumab, ranked in that order. However, none of these sequences resulted in maximum QALY gain. The finding that the ranking based on net benefit closely aligned with cost minimization and that no re-ranking occurred with lower cost per QALY thresholds suggests that differences in cost between TIMs are the major driver of rankings rather than differences in QALYs gained.

The QALY-maximizing approach to sequence optimization resulted in a small gain in incremental QALYs at substantially higher added costs. Absolute differences in the incremental QALYs gained vs conventional treatment for any sequence were small, ranging from 0.172 QALYs with ustekinumab-vedolizumab to 0.058 QALYs for golimumabadalimumab, with a difference of 0.113 .

In contrast, the differences in incremental cost were substantial,rangingfrom $\$ 166,094$ for ustekinumab-vedolizumab to $\$ 37,266$ for infliximab-dyyb-tofacitinib $(\triangle \$ 128,828)$. The QALY-maximizing sequence of ustekinumab-vedolizumab was not cost-effective compared with the NHB-maximizing sequence of infliximab-dyyb-tofacitinib. The differences for the QALY-maximizing sequence vs the highest NHB were 0.039 incremental QALYs and \$128,828 incremental costs. This yielded an incremental cost-effectiveness ratio of more than $\$ 3.3$ million, indicating that the QALYmaximizing sequence was far from cost-effective vs the highest NHB sequence.

Among subcutaneously administered TIMs, the sequence with the highest incremental NHB was golimumab-tofacitinib. Compared with the highest-ranking regimen in terms of incremental NHB (infliximab-dyyb-tofacitinib) overall, the more convenient subcutaneously administered regimen resulted in 0.044 fewer QALYs and \$27,668 higher costs, driven by a lack of less expensive biosimilar TIMs with subcutaneous administration. A NHB loss of 0.228 thus could be contributed to choosing a sequence with a more convenient mode of administration.

Strengths of our study are the robustness of efficacy parameters on which is it is based, arising from a systematic literature review and NMA conducted by ICER. In addition, the model structure and inputs were developed and refined throughout the ICER assessment of TIMs for the treatment of moderate to severe ulcerative colitis with input from patients, manufacturers, clinicians, and other stakeholders.

Previous studies of optimal sequencing of TIMs in ulcerative colitis generally align with our findings. Current treatment guidelines by the American Gastroenterological Association suggest that infliximab and vedolizumab may be preferred first-line therapy, with ustekinumab and tofacitinib as preferred second-line therapy. ${ }^{18}$ Our findings suggest that the sequencing of vedolizumab and ustekinumab could be flipped depending on the goals of treatment, with the sequence of vedolizumab followed by ustekinumab having the higher NHB, but the sequence of ustekinumab followed by vedolizumab having the greater QALYs. In a study of optimal vedolizumab place in therapy, vedolizumab was cost-effective as first-line treatment in a scenario where adalimumab was commonly used as first-line treatment, but not when infliximab was used as first line. ${ }^{25}$

Because FDA-approved labeling of tofacitinib limits its approved use to after failure of an anti-TNF inhibitor due to safety concerns, we did not consider tofacitinib as an option for first-line treatment. ${ }^{22} \mathrm{~A}$ cost-minimization analysis of up to 4 lines of treatment centered around the optimal place in therapy for tofacitinib found that tofacitinib was cost saving compared with adalimumab as first-line treatment in ulcerative colitis. ${ }^{26}$

The findings of our study may inform current practice and access to TIMs in several ways. Step therapy in ulcerative colitis may be supported in this case, given that no predictive biomarkers exist; small QALY differences are observed between agents; and costs differ substantially between agents. However, current step therapy sequences may not be aligned to maximum NHB or maximal QALYs. Adalimumab, a current market leader that can be used for multiple auto-immune disorders, ranks near the lowest when used as the first-line treatment of moderate to severe ulcerative colitis because of its relatively modest efficacy compared with other TIMs, coupled with relatively high cost. 
Formulary policies to "step through" adalimumab in ulcerative colitis are not supported by the findings of our study. In fact, adalimumab as first line was never ranked in the top 5 optimal sequences by any measure except in a scenario where it is followed by infliximab. However, this finding should be interpreted with caution, since the high failure rates with golimumab and adalimumab give an earlier transition to the next line of treatment with a more cost-effective option, infliximab.

Because of the lack of randomized controlled trials in the biologic-experienced population, efficacy data for infliximab as second-line treatment is based on data in the biologic-naive population-where TIMs often have higher efficacy compared with the biologic-experienced population. Evidence suggests that switching between TIMs with the same mechanism of action (eg, trial of 2 TNF- $\alpha$ antagonist) may result in worse outcomes than switching to a TIM with a different mechanism of action. Both of these factors would contribute to lower efficacy for infliximab as second-line treatment than assumed in our analysis. Thus, the NHB of sequences with infliximab as a secondline treatment may be overestimated.

Although one of the first TIMs approved for ulcerative colitis, infliximab still remains comparable in efficacy to newer TIMs at a lower cost, in part because of the availability of biosimilars. Our findings reinforce infliximab's position as a commonly used TIM for ulcerative colitis and demonstrate that the downward price pressure with the introduction of biosimilars may change optimal treatment sequencing.

\section{LIMITATIONS}

This study has some limitations to consider. Our model relied on the results of randomized controlled trials of TIMs in the biologic-naive and biologic-experienced populations. Not all TIMs have data in both populations, which limited our ability to investigate all possible sequences. In addition, the patient experience of ulcerative colitis is highly heterogeneous. Our study relied on average response and remission values obtained from clinical trials and did not intend to override individual patient-level selection of TIMs based on clinical judgement and shared decision making. We did not investigate treatment sequences of more than 2 TIMs.

For the purposes of our analysis, modeling additional lines of treatment would have reduced the relative differences between sequences of first and second TIMs, the treatment decisions of greatest interest. However, many patients will try and fail more than 2 TIMs. For these patients, having multiple treatment options is important, since all TIMs have been shown to be superior to conventional treatment.

\section{Conclusions}

Based on the results of this analysis, the optimal sequence of TIMs as measured by NHB and cost minimization was infliximab or biosimilars as first-line treatment, then moving to tofacitinib, adalimumab, or vedolizumab. Because efficacy across agents was relatively similar, results were driven by cost. Sequences that generated the most QALYs began with ustekinumab, followed by vedolizumab, tofacitinib, and adalimumab.

\section{DISCLOSURES}

This study was based on an evidence synthesis and economic evaluation sponsored by the Institute for Clinical and Economic Review (ICER). Pandey and Fazioli are employees of ICER.
Bloudek reports grants from ICER during the conduct of the study and personal fees from Astellas, Akcea, Dermira, GlaxoSmithKline, Sunovion, Seattle Genetics, and TerSera Therapeutics, outside the submitted work.

Pandey reports grants from California Healthcare Foundation, Harvard Pilgrim Healthcare, Kaiser Foundation Health Plan Inc., and the Donoghue Foundation, during the conduct of the study, and other support from Aetna, America's Health Insurance Plans, Anthem, AbbVie, Alnylam, AstraZeneca, Biogen, Genentech/Roche, GlaxoSmithSline, Harvard Pilgrim, Health Care Service Corporation, Health Partners, Johnson \& Johnson (Janssen), Kaiser Permanente, LEO Pharma, Mallinckrodt, Merck, Novartis, National Pharmaceutical Council, Premera, Prime Therapeutics, Regeneron, Sanofi, Spark Therapeutics, United Healthcare, HealthFirst, Pfizer, Boehringer-Ingelheim, uniQure, Evolve Pharmacy Solutions, and Humana, outside the submitted work.

Fazioli reports grants from Arnold Ventures, California Healthcare Foundation, Harvard Pilgrim Healthcare, Kaiser Foundation Health Plan Inc., and The Donaghue Foundation, during the conduct of the study, and other support from Aetna, America's Health Insurance Plans, Anthem, AbbVie, Alnylam, AstraZeneca, Biogen, Blue Shield of CA, Cambia Health Services, CVS, Editas, Express Scripts, Genentech/Roche, GlaxoSmithKline, Harvard Pilgrim, Health Care Service Corporation, Health Partners, Johnson \& Johnson (Janssen), Kaiser Permanente, LEO Pharma, Mallinckrodt, Merck, Novartis, National Pharmaceutical Council, Premera, Prime Therapeutics, Regeneron, Sanofi, Spark Therapeutics, United Healthcare, HealthFirst, Pfizer, Boehringer-lngelheim, uniQure, Evolve Phamacy Solutions, and Humana, outside the submitted work.

Ollendorf reports grants from ICER, during the conduct of the study, along with other support from CEA Registry sponsors and personal fees from EMD Serono, Amgen, Analysis Group, Aspen Institute/ University of Southern California, GalbraithWight, Cytokinetics, Sunovion, University of Colorado, Center for Global Development, and Neurocrine, outside the submitted work.

Carlson reports grants from ICER, during the conduct of the study, and personal fees from Allergan, outside the submitted work. 
The inputs and model framework that were leveraged for this analysis were presented as part of the ICER assessment of TIMs for the treatment of moderate to severe ulcerative colitis.

\section{REFERENCES}

1. Crohns \& Colitis Foundation.

Understanding ulcerative colitis. Accessed October 3, 2020. https://www.crohnsandcolitis.com/ulcerative-colitis

2. Fumery M, Singh S, Dulai PS, et al. Natural history of adult ulcerative colitis in population-based cohorts: a systematic review. Clin Gastroenterol Hepatol. 2018;16(3):343-56.

3. Bradley GM, Oliva-Hemker M. Pediatric ulcerative colitis: current treatment approaches including role of infliximab. Biologics. 2012;6:125-34.

4. Mayo Clinic. Ulcerative colitis. 2019. Accessed September 23, 2019. https:// www.mayoclinic.org/diseasesconditions/ ulcerative-colitis/symptoms-causes/ syc-20353326

5. Irvine EJ. Quality of life of patients with ulcerative colitis: past, present, and future. Inflamm Bowel Dis. 2008;14(4):554-65.

6. Kappelman KD, Rifas-Shiman SL, Porter C, et al. Direct health care costs of Crohn's disease and ulcerative colitis in United States children and adults. Gastroenterology. 2008;135(6):1907-13.

7. Ko CW, Singh S, Feuerstein JD, et al. AGA clinical practice guidelines on the management of mild-to-moderate ulcerative colitis. Gastroenterology. 2019;156(3):748-64.

8. Rubin DT, Ananthakrishnan AN, Siegel CA, Sauer BG, Long MD. ACG clinical guideline: ulcerative colitis in adults. Am J Gastroenterol. 2019;114(3):384-413.

9. Hoda KM, Collins JF, Knigge KL, Deveney KE. Predictors of pouchitis after ileal pouch-anal anastomosis: a retrospective review. Dis Colon Rectum. 2008;51(5):554-60.
10. Noda K, Okita Y, Mizutani Y, et al. Risk factors for arthropathy in patients with ulcerative colitis after total colectomy. Mod Rheumatol. 2021 Mar;31(2):468-73.

11. Barnes EL, Kochar B, Jessup HR, Herfarth HH. The incidence and definition of Crohn's disease of the pouch: a systematic review and meta-analysis. Inflamm Bowel Dis. 2019;25(9):1474-80.

12. Jarchin L, Spencer EA, Khaitov S, et al. De novo Crohn's disease of the pouch in children undergoing ileal pouch-anal anastomosis for ulcerative colitis. J Pediatr Gastroenterol Nutr. 2019;69(4):455-60.

13. Baker DM, Folan AM, Lee MJ, Jones GL, Brown SR, Lobo AJ. A systematic review and meta-analysis of outcomes after elective surgery for ulcerative colitis. Colorectal Dis. 2021;23(1):18-33.

14. Kayal M, Plietz M, Rizvi A, et al. Inflammatory pouch conditions are common after ileal pouch anal anastomosis in ulcerative colitis patients. Inflamm Bowel Dis. 2020;26(7):1079-86.

15. Institute for Clinical and Economic Review. Targeted immune modulators for ulcerative colitis: effectiveness and value. Final evidence report. October 16, 2020. Accessed October 24, 2020. https://icer. org/wp-content/uploads/2020/08/ ICER UC Final Evidence Report 101620.pdf

16. Wang C, Baer HM, Gaya DR, Nibbs RJB, Milling S. Can molecular stratification improve the treatment of inflammatory bowel disease? Pharmacol Res. 2019;148:104442.

17. Dalal SR, Cohen RD. What to do when biologic agents are not working in inflammatory bowel disease patients. Gastroenterol Hepatol (N Y). 2015;11(10):657-65.

18. American Gastroenterological Association. Pharmacological management of adult outpatients with moderate to severely active ulcerative colitis: clinical decision support tool. Gastroenterology. 2020;158(5):1462-63.
19. Paulden M. Calculating and interpreting ICERs and net benefit. Pharmacoeconomics. 2020;38(8):785-807.

20. National Institute for Health and Care Excellence. Single technology appraisal. Tildrakizumab for treating chronic plaque psoriasis after systemic therapy [ID1060]. Committee Papers. 2018. Accessed October 10, 2020. https://www. nice.org.uk/guidance/ta575/evidence/ appraisal-consultation-committeepapers-pdf-6772451437

21. National Institute for Health and Care Excellence. Single technology appraisal. Brodalumab for treating moderate to severe plaque psoriasis [ID878]. Committee Papers. 2018. Accessed October 10, 2020. https://www.nice. org.uk/guidance/ta511/documents/ committee-papers

22. Xeljanz (tofacitinib). Prescribing information. Pfizer; 2020. Accessed June 17, 2021. http://labeling.pfizer.com/ ShowLabeling.aspx?id=959

23. Ritter TE, Fourment C, Kuten SA, et al. Second-line biologic therapy after vedolizumab. Am J Gastroenterol. 2019;114:S429-S430.

24. Armuzzi A, daCosta DiBonaventura M, Tarallo M, et al. Treatment patterns among patients with moderate-to-severe ulcerative colitis in the United States and Europe. PLoS One. 2020;15(1):e0227914.

25. Scott FI, Luo M, Shah Y, et al. Identification of the most cost-effective position of vedolizumab among the available biologic drugs for the treatment of ulcerative colitis. J Crohns Colitis. 2020;14(5):575-87.

26. Milev S, daCosta DiBonaventura M, Quon P, et al. An economic evaluation of tofacitinib for the treatment of moderately-to-severely active ulcerative colitis: modeling the cost of treatment strategies in the United States. J Med Econ. 2019;22(9):859-68. 